\title{
Relación entre el consumo abusivo de alcohol y la violencia ejercida por el hombre contra su pareja en la unidad de valoración integral de violencia de género (UVIVG) de Sevilla
}

\author{
Relation between alcohol consumption and violence of men towards \\ their relationships in the UVIVG of Seville (Spain)
}

\begin{abstract}
Resumen
Se ha estudiado la posible relación entre el consumo de alcohol y la violencia ejercida por el hombre hacia la mujer con la que mantiene o ha mantenido relación sentimental, en una muestra de 50 parejas analizadas en la Unidad de Valoración Integral de Violencia de Género (UVIVG) de Sevilla, a lo largo de los años 2013 y 2014. Para ello se ha empleado una entrevista estructurada creada ad hoc, así como los cuestionarios MINI, MALT y CAGE. De las 50 parejas, en 25 , las mujeres que denunciaron afirmaban que sus parejas eran consumidoras de alcohol y en ocasiones de otras sustancias de adicción, diagnosticándose tal consumo únicamente en 16 de estos hombres; la otra mitad de la muestra negaba el consumo de sustancias de adicción en sus parejas. Los resultados obtenidos muestran que los hombres que consumen alcohol ejercen violencia hacia su pareja en mayor proporción en estado sobrio que en estado ebrio. No obstante, el consumo de alcohol supone un incremento añadido en la frecuencia de la violencia ejercida. Igualmente, los resultados del estudio ponen de manifiesto que los hombres que no consumen alcohol ejercen violencia en menor porcentaje que los que sí lo hacen.
\end{abstract}

Palabras clave: Consumo alcohol. Violencia en la pareja. Aumento de violencia.

\begin{abstract}
This article examines the possible relation between alcohol consumption and violence of men towards women with whom they share or have shared a relationship. Our sample is composed of 50 couples analyzed under the Comprehensive Assessment of Gender Violence Unit (UVIVG) of Seville (Spain), during 2013 and 2014. It has been used an structured interview which has been created ad hoc, as well as the MINI, MALT and CAGE questionnaires. In 25 of the 50 analyzed couples, the woman, who had denounced, declared that her partner consumed alcohol and that he often consumed other substances of abuse. Nevertheless, this consumption was only diagnosed in 16 of those men. The women of the other half of the sample denied any consumption of substances of abuse by their partners. The results obtained from the analyzed sample shows that men who consume alcohol are more violent towards their partners when they are sober, than when they are drunk. However, the alcohol consumption increases the frequency of violent actions. Moreover, the results of the analysis reveal that men who do not consume alcohol resort to violence in a lower percentage than those who consume alcohol.
\end{abstract}

Key words: Alcohol consumption. Gender violence. Violence increase.

\section{Llopis Giménez ${ }^{1}$} MI. Rodríguez García ${ }^{2}$ I. Hernández Mancha²

\section{${ }^{1}$ Médico Forense y}

Coordinadora de la UVIVG de Sevilla.

${ }^{2}$ Médico Forense de la UVIVG de Sevilla.

\section{Correspondencia:}

Celia Llopis Giménez

Avenida de la Buhaira 31,

$2^{a}$ planta

41018 Sevilla

E-mail: cellogim@hotmail.com

Fecha de recepción:

06.FEB.2015

Fecha de aceptación: 20.MAR.2015 


\section{Introducción}

\section{Alcoholismo, teorías y posibles consecuencias derivadas de él}

Según datos del Ministerio del Interior ${ }^{1}$, el alcoholismo, considerado como una dependencia o adicción al alcohol, en España, es un problema sociosanitario y económico grave, patente en nuestra sociedad actual, con una tasa de consumo del $10 \%$ para la población masculina y del $3 \%$ al $5 \%$ para la femenina. Hay que tener en cuenta que el consumo de alcohol no sólo va a ser perjudicial para la salud física y psíquica de la persona que lo consume, sino que también va a influir de manera negativa en su comportamiento con respecto a las personas que lo rodean (problemas legales, laborales, sociales, etc.), pudiendo ser un factor de riesgo que dé lugar a que se produzcan conductas violentas en general y hacia la pareja en particular. Las propias mujeres que denuncian haber sufrido malos tratos afirman, durante el reconocimiento médico forense realizado, que cuando sus parejas no se encuentran bajo los efectos del alcohol no sufren violencia alguna, cambiando la situación cuando dichos hombres han bebido. Es en tal afirmación en la que vamos a tratar de profundizar a lo largo del presente estudio.

Para poder determinar si una persona sufre un trastorno por consumo de alcohol, o consumo problemático, que va a suponerle un deterioro o malestar clínicamente significativo, debe presentar al menos dos de los siguientes criterios, según expone el DSM $V^{2}$ : tolerancia, abstinencia, deseo intenso de beber alcohol, ingerirlo en cantidades mayores o durante más tiempo del que inicialmente se pretendía, deseo persistente o esfuerzos para interrumpir tal consumo, empleo de mucho tiempo en actividades relacionadas con tal consumo, reducción de actividades sociales, incumplimiento de los deberes fundamentales, consumo en situaciones en las que provoca un daño físico y persistencia del consumo aun a sabiendas de los problemas que le causa a la persona que lo ingiere. Entre las manifestaciones clínicas más frecuentes relacionadas con el consumo de alcohol, citadas en el estudio de Riofrío y Rodríguez ${ }^{3}$, destacan cefalea, anorexia, astenia, hiperreflexia, pirosis retroesternal, dolor abdominal, diarrea, hipertensión arterial, temblor distal, etc. Los signos relacionados, evidentes durante la exploración física, en el caso de un consumo crónico serían facies pletórica, inyección conjuntival, arañas vasculares en la epidermis facial, fetor alcohólico, desnutrición, hipertrofia parotídea, eritema palmar, subictericia o ictericia, hepatomegalia, ginecomastia, obesidad y signos de tabaquismo importantes.
Del mismo modo, en dicho estudio se citan los síntomas y signos psiquiátricos más comunes, entre los que destacan agresividad, conducta irracional, discusiones, violencia, pérdida de memoria, insomnio, depresión, ansiedad, cuadros alucinatorios o delirantes, síndrome de abstinencia, delirium e intoxicaciones atípicas. Fals-Stewart et al. ${ }^{4}$, en la misma línea, valoran que las parejas que consumen tales sustancias tienen marcados déficits de comunicación, escasa capacidad de resolución de conflictos o de lidiar con situaciones estresantes, son agresivopasivos y carentes de compromiso, y proporcionan escaso apoyo emocional o comprensión a la pareja.

Entre las teorías que explicarían los efectos del consumo de alcohol cabe citar:

- La teoría de la desinhibición, la cual propone que las conductas agresivas, en condiciones normales, están controladas por mecanismos inhibidores. Cuando un sujeto consume alcohol, por su acción específica en el sistema nervioso central ocasiona desinhibición de la conducta y elimina cualquier control sobre los instintos e impulsos, lo que implica mayor dificultad para controlarlos, lo que a su vez favorece que puedan aflorar comportamientos agresivos. El sujeto va a tener la percepción de ser provocado y, ante ello, reacciona con violencia ${ }^{5}$.

- Por el contrario, la teoría del aprendizaje social aboga por que la relación entre la agresividad y el consumo de alcohol viene dada por la influencia del entorno sociocultural, el cual espera que se produzca dicha agresividad, y las propias expectativas del sujeto que lo consume ${ }^{6,7}$.

- En el estudio de Bell y Naugle ${ }^{8}$ se realiza una recopilación de algunas de las teorías que pueden verse implicadas en el empleo de la violencia contra la pareja, entre las cuales cabe citar lo que denominan "teorías individuales". Dentro de ellas se tienen en cuenta el contexto sociocultural del que proviene el sujeto y los antecedentes personales, entre los que figuran el consumo de alcohol y de otras sustancias de adicción (además de otros, como las características de personalidad, haber sufrido malos tratos en la infancia, etc.), que tratarían de aportar una explicación al empleo de la violencia en la pareja.

\section{Consumo de alcohol como factor de riesgo en el empleo de violencia por parte del hombre hacia la pareja}

Tras consultar diversa literatura al respecto, se comprueba que hay opiniones contrarias en lo que se 
refiere a la relación entre el consumo de alcohol y la violencia ejercida contra las mujeres. A continuación se comentan dichas opiniones opuestas.

- Por un lado, hay autores que, a pesar de que establecen una relación entre el consumo de alcohol y la violencia ejercida hacia la pareja, consideran que hay otra serie de factores que participan en el empleo de la violencia.

Margolin y Burman ${ }^{9}$, y Lee et al. ${ }^{10}$, citan ciertos factores de riesgo que pueden darse en los hombres para que se produzcan agresiones hacia sus parejas, como puede ser el consumo de alcohol o de otras sustancias de adicción, la escasa educación, el bajo estatus ocupacional (escaso salario), el desempleo, la baja asertividad, haber sufrido violencia de pequeños, el estrés, ciertos trastornos de la personalidad, actitudes de género, falta de capacidad de empatía y comunicación, etc. Pero a pesar de los estudios realizados, la literatura no responde a la pregunta del motivo por el cual algunos hombres, tras consumir alcohol, agreden a sus parejas y otros no, aunque se cumplan los factores de riesgo antes citados. Igualmente relacionan el consumo de alcohol continuado con mayores problemas psicológicos (como la depresión) y con mayor tendencia a emplear la violencia.

Keiley et al. ${ }^{11}$, y Stalans y Ritchie ${ }^{12}$, consideran el consumo de alcohol como un factor de riesgo, pero no aceptan que el alcohol pueda ser en sí mismo y él solo la causa directa de violencia familiar o de género. Argumentan que el comportamiento o conducta de un individuo bajo los efectos desinhibidores del alcohol va a ser similar al comportamiento del mismo individuo en estado sobrio, es decir, una persona que no es violenta no se transformará en violenta por encontrarse bajo los efectos del alcohol. Por el contrario, el que es violento sin haber bebido seguirá siéndolo, o aumentará su violencia cuando beba. Valoran, por tanto, que el consumo de alcohol puede suponer un incremento en la gravedad o de la frecuencia del empleo de la violencia en la pareja en ambos, con mayor frecuencia por parte del hombre hacia la mujer.

Lorente y Toquero de la Torre ${ }^{13}$ añaden a lo anterior que es una excusa que emplean tanto el agresor como la víctima para justificar su conducta, y que no sólo se produce agresividad por la acción fisiológica de desinhibición que ejerce el alcohol sobre el organismo, sino también por factores ambientales (individuales, sociales y situacionales) en los que se desenvuelven estos individuos (inestabilidad laboral, poca experien- cia laboral, bajo nivel educativo, problemas de salud física y mental, problemas personales o legales...). No obstante, Lipsky et al. ${ }^{14}$ resaltan en su estudio la importancia de realizar tratamientos de desintoxicación-deshabituación, junto con terapia de pareja; lo que valoran que reduce tanto los consumos como las conductas violentas.

Oberleitner et al. ${ }^{15}$ añaden un elemento más a tener en cuenta en la actitud violenta, que sería la ira, a la que definen como "la forma de percibir, interpretar y responder a determinados eventos que suscitan una reacción emocional". Basándose en lo anterior, consideran que el tratamiento que reduce la ira puede igualmente implicar una reducción en el comportamiento violento. Relacionan la presencia de ira elevada con consumo de alcohol y agresividad en los hombres que agreden a sus parejas, y afirman que los hombres que ejercen violencia contra sus parejas y que presentan elevada ira son más propensos a reaccionar con agresividad cuando consumen alcohol. Consideran, por tanto, que el alcohol incrementa o exacerba la violencia, produciéndose episodios de mayor intensidad de esta cuando se consume alcohol que cuando no se consume. Afirman que el alcohol está relacionado con el $40 \%$ al $60 \%$ de los casos de violencia hacia la pareja.

- Hay autores, los mínimos, que establecen una relación directa entre consumo de alcohol y agresividad. Son pocos los autores, destacando Bennett ${ }^{16}$, y Hines y Douglas ${ }^{17}$, que afirman que los hombres violentos muestran mayor impulsividad e ira, y presentan mayor consumo de alcohol y otras sustancias de abuso, que los hombres no violentos, haciendo responsables directas a dichas sustancias de abuso de la destrucción del sistema familiar. Por tanto, relacionan directamente el consumo de alcohol (agudo o crónico) y otras sustancias de adicción por parte del hombre con la violencia ejercida hacia su pareja, y afirman que un nivel elevado de alcoholismo predice la conducta violenta.

Estudios realizados fuera del ámbito carcelario, como el de Kantor y Straus ${ }^{18}$, valoran que los hombres que ejercen violencia hacia sus parejas son más propensos a consumir alcohol y drogas de manera habitual, y que en el momento de cometerse una agresión, aproximadamente en el $15 \%$ al $20 \%$ de los casos uno de los dos miembros de la pareja o ambos han bebido alcohol, y un $8 \%$ han consumido drogas.

Bennett ${ }^{16}$ recoge en su estudio que es ocho veces más probable que se produzca un episodio 
violento un día en que un hombre ha consumido alcohol que un día en que ese mismo hombre no lo ha hecho. Se demuestra que existe tal relación durante las intervenciones, de manera que si una persona que consume sustancias de adicción y agrede a su pareja sigue un tratamiento de desintoxicación, se va a producir una disminución tanto en sus consumos como en la violencia.

- Entre los autores que no establecen tal relación, destacan Miller ${ }^{19}$ y Testa et al. ${ }^{20}$, quienes abogan por una postura contraria y no consideran el consumo de alcohol como un factor favorecedor del empleo de la violencia, sino al contrario, consideran que el empleo de violencia en la pareja contribuye al consumo de alcohol, tanto en el agresor como en la víctima. Añaden que el consumo de alcohol en una mujer no es un factor predictor de sufrir violencia de género. Sin embargo, el hecho de sufrir dicha violencia si es un factor predictor de poder padecer ansiedad y depresión, así como de consumir alcohol (y otras sustancias de adicción) en un futuro, entendido como una forma de deseo de disminuir el efecto negativo del maltrato sufrido y buscar alivio o refugio en él (consecuencia de dicha violencia). Hirschel y Hutchison ${ }^{21}$ afirman en su estudio que hay una tendencia popular a pensar que la violencia en la pareja es siempre perpetrada hacia una mujer por un hombre generalmente bajo los efectos del alcohol o de las drogas, excusando su conducta en tales consumos. Consideran que la violencia en la pareja incluye posibilidades como que sea la mujer la causante de esta, o que se perpetre entre parejas del mismo sexo, y no consideran que el alcohol sea un agente causante de tal violencia. No obstante, centran su estudio en los casos de violencia perpetrada por un varón hacia su pareja mujer, y valoran que en la mayoría de estos casos no está presente el consumo de alcohol o de sustancias de adicción.

\section{Importancia del consumo de alcohol por parte de la mujer}

Aunque la mayoría de los estudios hablan del consumo de alcohol por parte del hombre relacionado con la violencia ejercida hacia la mujer, muy pocos han investigado la posible relación entre el consumo de alcohol y la conducta violenta de la mujer hacia su pareja, mostrando resultado negativo o sin resultado evidente en su gran mayoría. Destacan los estudios de Cunradi et al. ${ }^{22}$, Klostermann y Falas-Stewart ${ }^{23}$, y Leonard ${ }^{24}$, que ponen de manifiesto que en el caso de que sea la mujer la que beba no se establece relación entre el consumo de alcohol y el empleo de agresividad unidireccional (por parte de uno de los dos miembros de la pareja) o bidireccional, y que la evidencia a la hora de ejercer violencia en la mujer es menor que cuando es el hombre quien bebe. Si es el hombre el que consume alcohol, los resultados son contrarios y sí se asocia dicho consumo con el empleo de violencia, tanto hacia su pareja como de manera mutua. Caetano et al. ${ }^{25}$ son de los pocos autores que relacionan el consumo de alcohol por parte de la mujer con el empleo de violencia bidireccional.

Autores como Downs et al. ${ }^{26}$, Kantor y Straus ${ }^{18}$, y Miller ${ }^{19}$, afirman que también es importante tener en cuenta el consumo de alcohol por parte de las mujeres víctimas de violencia ejercida por los hombres (consumidores y no consumidores), ya que tal consumo supone a su vez un aumento de violencia en la pareja.

Con lo anteriormente expuesto queda patente que el consumo de sustancias de adicción y la agresión hacia la pareja se producen en muchas ocasiones de manera conjunta.

\section{Objetivos e hipótesis}

En el presente estudio se ha planteado alcanzar los siguientes objetivos:

- Determinar las posibles diferencias existentes en la historia de malos tratos entre los hombres cuyas parejas afirman de ellos un consumo de alcohol y aquellos de los que sus parejas niegan tal consumo de alcohol.

- Valorar la relación entre el consumo de alcohol y el empleo de violencia.

Se plantean una serie de hipótesis:

- Los hombres denunciados por malos tratos y que consumen alcohol, en comparación con los que no lo consumen, emplean violencia contra sus parejas en mayor medida.

- Los hombres violentos que consumen alcohol, cuando no se encuentran bajo los efectos de dicha sustancia no ejercen violencia; por el contrario, sí la ejercen cuando han bebido.

- Los hombres violentos que no consumen alcohol emplean violencia contra sus parejas en menor medida que aquellos hombres igualmente violentos y que sí beben.

\section{Material y método}

El presente trabajo se centra en establecer una posible relación entre el consumo de alcohol y el hecho 
de ejercer violencia por parte del hombre hacia su pareja. Para ello se ha llevado a cabo un estudio en 50 parejas, en las que las mujeres han interpuesto denuncia por malos tratos y han sido reconocidas en la Unidad de Valoración Integral de Violencia de Género (UVIVG) de Sevilla. La muestra se ha obtenido a lo largo de los años 2013 y 2014 de manera secuencial, a medida que las parejas iban acudiendo a la UVIVG.

Se han obtenido así dos grupos muestrales: el grupo 1 , constituido por los hombres cuyas parejas afirman de ellos que consumen alcohol, independientemente de que con posterioridad se haya valorado la existencia o no de tal consumo, y el grupo 2 o grupo control, formado por parejas en las que el consumo de alcohol no ha estado presente en su relación, o ha sido mínimo y no ha supuesto problema alguno en la relación. En ambos grupos se ha estudiado fundamentalmente al hombre. El estudio de la mujer se ha centrado en la historia de malos tratos denunciada y en si se establece relación alguna entre el consumo de alcohol y el empleo de violencia por parte de sus parejas.

Tras informar de manera verbal y por escrito de la finalidad de nuestro estudio a cada persona explorada, se obtuvo el consentimiento informado por escrito de todos los participantes. Entre los criterios de inclusión figuran: a) ser mayor de 18 años; b) haber denunciado o haber sido denunciado por su pareja o ex pareja por malos tratos; c) que ambos miembros de la pareja acudan a valoración a la UVIVG de Sevilla; d) que ambas partes de la pareja tengan un nivel cultural suficiente y comprensión adecuada del idioma español para poder contestar a las preguntas y realizar las pruebas pertinentes; e) acceder de manera voluntaria a la participación en el estudio tras haber sido debidamente informados; y f) los sujetos del grupo 1, para su inclusión, tenían que haber sido acusados de consumo de alcohol por parte de sus parejas en la declaración. Como criterio de exclusión sólo se consideró la existencia de alteraciones mentales graves o la ausencia de nivel cultural suficiente para la comprensión de las pruebas.

La dinámica de exploración consistió en examinar de forma separada a la denunciante y al imputado, empleando para ello una entrevista estructurada creada ad hoc. Esta entrevista se centra fundamentalmente en el estudio de los datos sociodemográficos, datos de la familia de referencia, infancia, adolescencia, datos relativos a las anteriores relaciones de pareja, datos sobre la relación de pareja entre la denunciante y el denunciado, historia de malos tratos, estado de salud, consumo de sustancias adictivas y posibles denuncias interpuestas. Posteriormente, en el caso del hombre, se realizaba una pequeña exploración física para valorar cualquier síntoma o signo indicativo de posible consumo de alcohol (temblor de manos, halitosis alcohólica, facies abotargada, lengua característica, alteración de marcadores hepáticos en una analítica, etc.). Por último, a los imputados se les aplicaron tres cuestionarios: MINI (herramienta de ayuda para realizar posibles diagnósticos de consumo de alcohol), MALT (útil en la detección de sujetos con dependencia alcohólica y problemas de alcoholismo, y en la detección de pacientes con un alto grado de negación) y CAGE (método de cribado para detectar un posible abuso de alcohol).

\section{Resultados obtenidos en la entrevista}

\section{Análisis descriptivo y comparativo de los datos sociodemográficos}

En la Tabla 1 se observa que el grupo de hombres acusados de consumo de alcohol está constituido por 25 sujetos con edades comprendidas entre los 27 y los 72 años, con una media de 49,5 años. Cerca de la mitad (52\%) se encuentran divorciados, separados o en trámites de separación o divorcio, y tan sólo un $24 \%$ están casados. Predominan los hombres con nivel de estudios primarios $(44,4 \%)$ y la mayoría se encuentran en situación de desempleo (60\%). Aproximadamente la mitad de la muestra viven solos (44\%) y se consideran independientes económicamente un tercio de ellos, a pesar de la alta tasa de desempleo.

En el grupo consumo se concluye que ha habido una situación compatible con violencia de género o malos tratos en 10 de los 25 casos estudiados. En los 15 casos restantes se valora que se ha producido una situación compatible con conflicto de pareja.

El grupo 2, o grupo control (hombres que no consumen alcohol), está constituido por 25 hombres con edades comprendidas entre los 19 y los 62 años, con una media de 40,5 años. Tres cuartos de la muestra (80\%) se encuentran divorciados, separados o en trámites de separación o divorcio, y tan sólo un $12 \%$ están casados. Predominan los hombres con nivel de estudios primarios (36\%). La mayoría se encuentran en situación laboral activa (64\%) y se consideran económicamente independientes en el mismo porcentaje, a pesar de lo cual algo más de la mitad de la muestra viven con sus padres $u$ otros familiares (56\%), a diferencia de lo descrito en el grupo anterior.

En el grupo control se concluye que ha habido violencia de género o malos tratos en 6 de los 25 casos estudiados. En los 19 restantes se valora que se ha producido una situación compatible con conflicto de pareja o ausencia de violencia de género. 


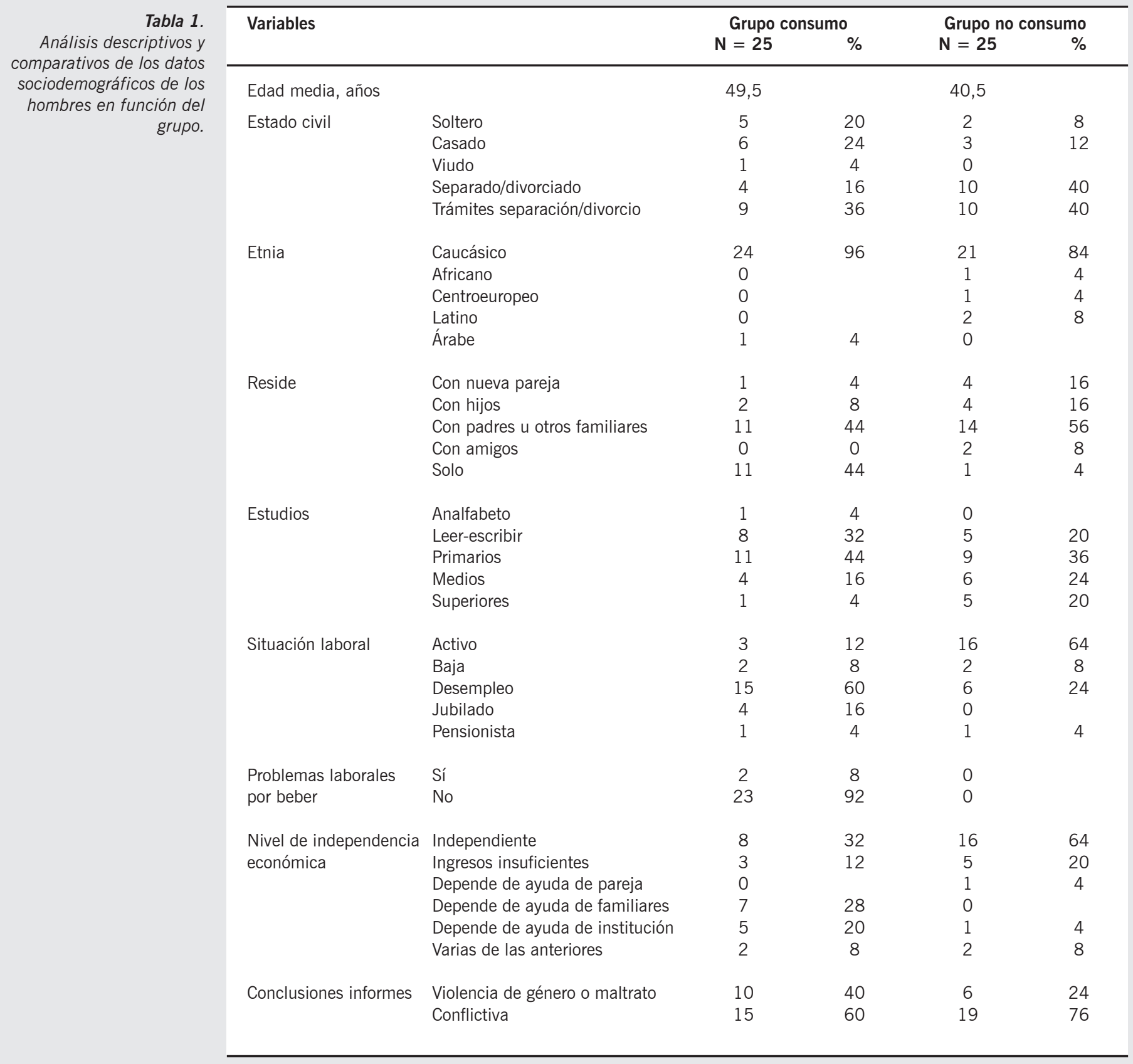

\section{Análisis de los datos relacionados con la familia de referencia y con anteriores relaciones de pareja}

En la Tabla 2 se observa que los hombres de ambos grupos afirman, en su mayoría, mantener una buena relación con su padre y hermanos, y en su totalidad con su madre. Destaca que dos hombres de ambos grupos ( $8 \%$ ) afirman que sus padres les maltrataban. Igualmente, cinco hombres del grupo de consumo (20\%) y uno del grupo control (4\%) reconocen haber presenciado conductas de malos tratos por parte de 


\begin{tabular}{|c|c|c|c|c|c|}
\hline \multirow[t]{2}{*}{ Variables } & & \multicolumn{2}{|c|}{ Grupo consumo } & \multicolumn{2}{|c|}{ Grupo no consumo } \\
\hline & & $N=25$ & $\%$ & $N=25$ & $\%$ \\
\hline \multirow[t]{2}{*}{ Padres separados } & No & 21 & 84 & 22 & 88 \\
\hline & Sí & 4 & 16 & 3 & 12 \\
\hline \multirow[t]{3}{*}{ Relación buena con } & Padre & 23 & 92 & 23 & 92 \\
\hline & Madre & 25 & 100 & 25 & 100 \\
\hline & Hermanos & 23 & 92 & 23 & 92 \\
\hline \multirow[t]{2}{*}{ Si el padre del denunciado maltrataba a } & Denunciado & 2 & 8 & 2 & 8 \\
\hline & Madre & 5 & 20 & 1 & 4 \\
\hline \multirow[t]{2}{*}{ Padre } & Bebe en exceso & 7 & 28 & 2 & 8 \\
\hline & Consume drogas & 1 & 42 & 0 & \\
\hline \multirow[t]{2}{*}{ Madre } & Bebe en exceso & 1 & 4 & 0 & \\
\hline & Consume drogas & 0 & & 0 & \\
\hline \multirow[t]{2}{*}{ Familiar } & Bebe en exceso & 3 & 12 & 0 & \\
\hline & Consume drogas & 1 & 4 & 0 & \\
\hline \multirow[t]{3}{*}{ Relaciones previas a la denunciante } & Sin convivencia & 4 & 16 & 5 & 20 \\
\hline & Con convivencia & 12 & 48 & 5 & 20 \\
\hline & Existencia de maltrato & 0 & & 0 & \\
\hline \multirow[t]{2}{*}{ Con respecto a nueva pareja } & Si la tiene & 4 & 16 & 10 & 40 \\
\hline & Si vive con ella & 1 & 4 & 1 & 4 \\
\hline \multirow[t]{2}{*}{ Con respecto a la denunciante } & Mantienen actual relación & 2 & 8 & 0 & \\
\hline & Vive con ella & 1 & 4 & 0 & \\
\hline
\end{tabular}

su padre hacia su madre. En el grupo de consumo de alcohol, cerca de un tercio de la muestra admite que su padre bebe, y uno de ellos refiere que es la madre la que bebe alcohol. Estas cifras disminuyen considerablemente en el caso de los hombres del grupo control.

Destaca el superior porcentaje de hombres del grupo control, frente al grupo consumo, que mantienen una nueva relación de pareja. Igualmente resulta destacable el hecho de que, tras la denuncia, dos hombres del grupo de consumo continúan manteniendo una relación sentimental con su pareja, y que incluso uno de ellos continúa conviviendo con ella.

\section{Análisis de los datos sobre la historia de malos tratos}

La Tabla 3 muestra que es similar el porcentaje tanto de hombres como de mujeres en ambos grupos que
Tabla 2.

Análisis descriptivos y comparativos de los datos de la familia de referencia y de anteriores relaciones de pareja de los hombres en función del grupo. reconocen que discutían durante su relación, siendo significativa la diferencia entre ambos grupos en lo que respecta a las discusiones por celos o consumo de alcohol. Según lo referido por las denunciantes durante las entrevistas, los principales motivos de tales discusiones en el grupo de consumo son el dinero, la intromisión de la familia, la actitud de la pareja o los celos mostrados por esta, y el consumo de alcohol. Destaca el porcentaje de hombres de ambos grupos (cerca de un tercio de la muestra) que admiten ser celosos, en contraposición de lo que afirman sus parejas sobre ellos, las cuales valoran dicho porcentaje como superior. Las mujeres se consideran celosas en un porcentaje inferior, y sin embargo tanto hombres como mujeres sospechan infidelidad en sus parejas en un $30 \%$.

Los miembros de la pareja de ambos grupos admiten, en su gran mayoría, que los hijos estaban presentes durante las discusiones o problemas en la pareja. 


\begin{tabular}{|c|c|c|c|c|c|c|}
\hline \multirow{2}{*}{$\begin{array}{r}\text { Tabla } 3 . \\
\text { Análisis descriptivos y } \\
\text { comparativos de la historia } \\
\text { de malos tratos según lo } \\
\text { referido por ambos según el } \\
\text { grupo. }\end{array}$} & \multirow[t]{2}{*}{ Variables } & & \multicolumn{2}{|c|}{ Grupo consumo } & \multicolumn{2}{|c|}{ Grupo no consumo } \\
\hline & & & $\begin{array}{c}\text { Hombre } \\
\mathrm{N}=25(\%)\end{array}$ & $\begin{array}{c}\text { Mujer } \\
\mathrm{N}=25(\%)\end{array}$ & $\begin{array}{c}\text { Hombre } \\
\mathrm{N}=25(\%)\end{array}$ & $\begin{array}{c}\text { Mujer } \\
\mathrm{N}=25(\%)\end{array}$ \\
\hline & Discusiones por & $\begin{array}{l}\text { Hijos } \\
\text { Dinero } \\
\text { Trabajo } \\
\text { Ocio } \\
\text { Amigos } \\
\text { Sexo } \\
\text { Tareas del hogar } \\
\text { Familia extensa } \\
\text { Actitud pareja/celos } \\
\text { Consumo alcohol de él }\end{array}$ & $\begin{array}{l}13(52) \\
11(44) \\
10(40) \\
11(44) \\
4(16) \\
6(24) \\
4(16) \\
8(32) \\
14(56) \\
4(16)\end{array}$ & $\begin{array}{l}15(60) \\
21(84) \\
11(44) \\
14(56) \\
12(48) \\
12(48) \\
10(40) \\
15(60) \\
19(76) \\
14(56)\end{array}$ & $\begin{array}{l}2(8) \\
2(8) \\
1(4) \\
0 \\
0 \\
1(4) \\
1(4) \\
2(8) \\
2(8) \\
2(8)\end{array}$ & $\begin{array}{l}1(4) \\
3(12) \\
3(12) \\
0 \\
1(4) \\
1(4) \\
1(4) \\
1(4) \\
7(28) \\
0\end{array}$ \\
\hline & Problemas por ser celoso & $\begin{array}{l}\text { No, nunca } \\
\text { En ocasiones } \\
\text { Con frecuencia }\end{array}$ & $\begin{array}{l}17(68) \\
5(20) \\
3(12)\end{array}$ & $\begin{array}{c}24(96) \\
1(4) \\
0\end{array}$ & $\begin{array}{c}18(72) \\
7(28) \\
0\end{array}$ & $\begin{array}{c}23(92) \\
2(8) \\
0\end{array}$ \\
\hline & Si su pareja es celosa & $\begin{array}{l}\text { No } \\
\text { Sólo un poco } \\
\text { Sí }\end{array}$ & $\begin{aligned} 1 & (44) \\
1 & (4) \\
13 & (52)\end{aligned}$ & $\begin{array}{c}3(12) \\
5(20) \\
17(68)\end{array}$ & $\begin{array}{c}16(62) \\
2(8) \\
7(28)\end{array}$ & $\begin{array}{c}10(40) \\
0 \\
15(60)\end{array}$ \\
\hline & $\begin{array}{l}\text { Celos de su pareja } \\
\text { dan problemas }\end{array}$ & $\begin{array}{l}\text { No, nunca } \\
\text { En ocasiones } \\
\text { Con frecuencia }\end{array}$ & $\begin{array}{l}12(52) \\
4(16) \\
8(32)\end{array}$ & $\begin{array}{c}6(24) \\
6(24) \\
13(52)\end{array}$ & $\begin{array}{l}17(68) \\
3(12) \\
5(20)\end{array}$ & $\begin{array}{c}10(40) \\
5(20) \\
10(40)\end{array}$ \\
\hline & Hijos presentes & $\begin{array}{l}\text { Nunca } \\
\text { En ocasiones } \\
\text { Con frecuencia }\end{array}$ & $\begin{array}{c}5(20) \\
17(68) \\
3(12)\end{array}$ & $\begin{array}{c}4(16) \\
9(36) \\
12(48)\end{array}$ & $\begin{array}{c}7(28) \\
18(72) \\
0\end{array}$ & $\begin{array}{c}2(8) \\
14(56) \\
9(36)\end{array}$ \\
\hline & Sufre maltrato & $\begin{array}{l}\text { Sí } \\
\text { No }\end{array}$ & $\begin{array}{c}7(28) \\
18(72)\end{array}$ & $\begin{array}{c}25(100) \\
0\end{array}$ & $\begin{array}{c}9(36) \\
16(64)\end{array}$ & $\begin{array}{c}25(100) \\
0\end{array}$ \\
\hline & $\begin{array}{l}\text { Conductas de malos } \\
\text { tratos ejercidas por } \\
\text { denunciado hacia } \\
\text { denunciante, según } \\
\text { lo referido por ambos }\end{array}$ & $\begin{array}{l}\text { Le gritaba } \\
\text { Le intimidaba } \\
\text { Le humillaba } \\
\text { Le insultaba } \\
\text { Le amenazaba } \\
\text { Le pegaba con las manos } \\
\text { Le pegaba con objetos } \\
\text { Rompía cosas } \\
\text { Chantaje emocional }\end{array}$ & $\begin{array}{c}19(76) \\
2(8) \\
4(16) \\
11(44) \\
2(8) \\
1(4) \\
0 \\
8 \\
2(8)\end{array}$ & $\begin{array}{l}25(100) \\
21(84) \\
24(96) \\
25(100) \\
21(84) \\
16(64) \\
5(20) \\
21(84) \\
21(84)\end{array}$ & $\begin{array}{c}10(40) \\
2(8) \\
3(12) \\
10(40) \\
1(4) \\
1(4) \\
0 \\
0 \\
0\end{array}$ & $\begin{array}{l}21(84) \\
15(60) \\
16(64) \\
23(92) \\
12(48) \\
18(72) \\
3(12) \\
7(28) \\
15(60)\end{array}$ \\
\hline
\end{tabular}




\begin{tabular}{|c|c|c|c|c|c|}
\hline \multirow[t]{3}{*}{ Variables } & & \multirow{2}{*}{\multicolumn{2}{|c|}{ Grupo consumo }} & \multirow{2}{*}{\multicolumn{2}{|c|}{ Grupo no consumo }} \\
\hline & & & & & \\
\hline & & $\mathrm{N}=25(\%)$ & $N=25(\%)$ & $\mathrm{N}=25(\%)$ & $\mathrm{N}=25(\%)$ \\
\hline & Aislamiento social & $3(12)$ & $17(68)$ & $2(8)$ & $18(72)$ \\
\hline & Aislamiento familiar & $3(12)$ & $16(64)$ & 0 & $13(52)$ \\
\hline & Restricción económica & $1(4)$ & $10(40)$ & 0 & $12(48)$ \\
\hline & Abuso sexual con coacción & 0 & $10(40)$ & 0 & $8(32)$ \\
\hline & Abuso sexual con fuerza & 0 & $3(12)$ & 0 & $2(8)$ \\
\hline & Acoso & 0 & $12(48)$ & 0 & $10(40)$ \\
\hline & Lloraba & $3(12)$ & $17(68)$ & 0 & $9(36)$ \\
\hline \multirow{4}{*}{$\begin{array}{l}\text { Violencia ejercida por } \\
\text { denunciante al imputado }\end{array}$} & Le gritaba & $23(92)$ & $20(80)$ & $16(64)$ & $9(36)$ \\
\hline & Le insultaba/humillaba & $22(88)$ & $14(56)$ & $18(72)$ & $10(40)$ \\
\hline & Le amenazaba & $10(40)$ & $5(20)$ & $6(24)$ & $2(8)$ \\
\hline & Le pegaba & $8(32)$ & $6(24)$ & $4(16)$ & $4(16)$ \\
\hline \multirow{4}{*}{$\begin{array}{l}\text { Tipo de violencia } \\
\text { más frecuente }\end{array}$} & Física & $1(4)$ & $2(8)$ & $1(4)$ & $2(8)$ \\
\hline & Psicológica & $14(56)$ & $22(88)$ & $12(48)$ & $23(92)$ \\
\hline & Sexual & 0 & $1(4)$ & 0 & 0 \\
\hline & Ninguna & $10(40)$ & 0 & $12(48)$ & 0 \\
\hline \multirow{3}{*}{$\begin{array}{l}\text { Agresiones a familiares } \\
\text { por el imputado }\end{array}$} & No, nunca & $18(72)$ & $10(40)$ & $24(96)$ & $16(64)$ \\
\hline & En ocasiones & $4(16)$ & $6(24)$ & $1(4)$ & $4(16)$ \\
\hline & Con frecuencia & $3(12)$ & $9(36)$ & 0 & $5(20)$ \\
\hline \multirow{3}{*}{$\begin{array}{l}\text { Agresiones a otros } \\
\text { por el imputado }\end{array}$} & No, nunca & $20(80)$ & $11(44)$ & $22(88)$ & $16(64)$ \\
\hline & En ocasiones & $3(12)$ & $9(36)$ & $3(12)$ & $5(20)$ \\
\hline & Con frecuencia & $2(8)$ & $5(20)$ & 0 & $4(16)$ \\
\hline \multirow{2}{*}{$\begin{array}{l}\text { El denunciado } \\
\text { tenía armas }\end{array}$} & Sí & $1(4)$ & $3(12)$ & 0 & $3(12)$ \\
\hline & No & $24(96)$ & $22(88)$ & $25(100)$ & 22 (88) \\
\hline
\end{tabular}

Tabla 3.

(continuación).
En lo que respecta a la historia de malos tratos, resulta destacable el escaso número de hombres que admiten conductas de violencia hacia sus parejas, en especial las referentes a agresiones físicas y sexuales. Destaca igualmente que es mayor el número de mujeres del grupo de consumo que afirman que sufren malos tratos verbales en mayor medida, así como físicos y sexuales.

Resulta sorprendente que un tercio de los hombres de ambos grupos considera que sufre malos tratos. Las mujeres del grupo de consumo admiten en un $56 \%$ que respondían con violencia verbal hacia sus parejas, con amenazas o con agresiones físicas en un $20 \%$ respectivamente. Este porcentaje desciende considerablemente en el grupo control.

Un cierto porcentaje de hombres del grupo de consumo reconoce haber ejercido violencia hacia otros miembros de su familia.
En la Tabla 4 destaca que es mayor el porcentaje de conductas de violencia ejercidas por los hombres del grupo de consumo cuando no beben, fundamentalmente las conductas de violencia psíquica, a diferencia de lo que refieren sus parejas en un primer momento (antes de analizar cada conducta por separado). El hecho de beber supone que dichas conductas se sigan produciendo, sumándose a las que ya habían ocurrido en estado sobrio.

\section{Análisis de los datos sobre el estado de salud y los antecedentes penales}

En la Tabla 5 puede verse que un $32 \%$ de los hombres del grupo de consumo afirma tener una enfermedad física en el momento de la entrevista, frente a un $20 \%$ del grupo control. Del mismo modo, es superior el porcentaje de hombres del grupo de consumo que 
Tabla 4

Análisis descriptivos y comparativos de la historia de malos tratos según lo referido por la denunciante en función del grupo y del consumo o no de alcohol.

\section{Variables}

Grupo consumo

Cuando no bebe Sólo cuando bebe

$\mathrm{N}=25(\%) \quad \mathrm{N}=25(\%)$

Grupo no consumo

$\mathrm{N}=25(\%)$

Conductas de malos tratos

$\begin{array}{lc}\text { Le gritaba } & 16(64) \\ \text { Le intimidaba } & 8(32) \\ \text { Le humillaba } & 13(52) \\ \text { Le insultaba } & 14(56) \\ \text { Le amenazaba } & 10(40) \\ \text { Le pegaba con manos } & 9(36) \\ \text { Le pegaba con objetos } & 4(16) \\ \text { Rompía cosas } & 13(52) \\ \text { Chantaje emocional } & 13(52) \\ \text { Aislamiento social } & 13(52) \\ \text { Aislamiento familiar } & 14(56) \\ \text { Restricción económica } & 9(36) \\ \text { Abuso sexual con coacción } & 8(32) \\ \text { Abuso sexual con fuerza } & 2(8) \\ \text { Acoso } & 10(40)\end{array}$

9 (36)

$21(84)$

$15(60)$

$16(64)$

$23(92)$

$12(48)$

18 (72)

3 (12)

7 (28)

$15(60)$

18 (72)

$13(52)$

$12(48)$

8 (32)

2 (8)

$10(40)$

Tabla 5.

Análisis descriptivos y comparativos de los datos sobre el estado de salud y los antecedentes penales de los hombres según el grupo.

\begin{tabular}{|c|c|c|c|c|c|}
\hline \multirow[t]{2}{*}{ Variables } & & \multicolumn{2}{|c|}{ Grupo consumo } & \multicolumn{2}{|c|}{ Grupo no consumo } \\
\hline & & $N=25$ & $\%$ & $N=25$ & $\%$ \\
\hline Enfermedad física actual & & 8 & 32 & 5 & 20 \\
\hline Enfermedad física en el pasado & & 7 & 28 & 7 & 28 \\
\hline Ha ido al psiquiatra o psicólogo & & 9 & 36 & 5 & 20 \\
\hline Va al psiquiatra o psicólogo ahora & & 2 & 8 & 3 & 12 \\
\hline Le han prescrito tratamiento antes & & 10 & 40 & 5 & 20 \\
\hline Le han prescrito tratamiento ahora & & 13 & 52 & 3 & 12 \\
\hline Enfermedad mental familiares & & 3 & 12 & 1 & 4 \\
\hline Ha estado hospitalizado & & 15 & 60 & 6 & 24 \\
\hline $\begin{array}{l}\text { ¿Le han denunciado por ejercer } \\
\text { violencia contra su pareja? }\end{array}$ & $\begin{array}{l}\text { Sí } \\
\text { No }\end{array}$ & $\begin{array}{c}22 \\
3\end{array}$ & $\begin{array}{l}88 \\
12\end{array}$ & $\begin{array}{c}25 \\
0\end{array}$ & 0 \\
\hline ¿Ha denunciado a su pareja? & $\begin{array}{l}\text { Sí } \\
\text { No }\end{array}$ & $\begin{array}{c}3 \\
22\end{array}$ & $\begin{array}{l}12 \\
88\end{array}$ & $\begin{array}{c}1 \\
24\end{array}$ & $\begin{array}{c}4 \\
96\end{array}$ \\
\hline ¿Ha retirado ella la denuncia? & $\begin{array}{l}\text { Sí } \\
\text { No }\end{array}$ & $\begin{array}{c}6 \\
19\end{array}$ & $\begin{array}{l}24 \\
76\end{array}$ & $\begin{array}{c}0 \\
25\end{array}$ & 100 \\
\hline
\end{tabular}


ha acudido al psicólogo o al psiquiatra y que toman o han tomado psicofármacos.

Destaca el porcentaje de hombres del grupo de consumo que ha denunciado a su pareja (12\%), así como el porcentaje de denuncias retiradas en dicho grupo (24\%). Igualmente hay que destacar que es superior el porcentaje de hombres del grupo de consumo (32\%), frente al grupo control (12\%), que presenta antecedentes penales previos y orden de alejamiento de su pareja (48\% frente a $8 \%$, respectivamente).

\section{Análisis de los datos sobre el consumo de sustancias adictivas}

En la Tabla 6 resulta destacable el porcentaje de hombres del grupo de consumo que admiten su consumo de alcohol (96\%), a pesar de que únicamente un 32\% admite que el consumo es elevado o que supone un problema para su salud. Algo más de un $60 \%$ admite que se emborracha, y un $24 \%$ reconoce que se torna violento cuando ello ocurre. Sólo un $20 \%$ acuden a deshabituación-desintoxicación.

\begin{tabular}{|c|c|c|c|c|c|}
\hline \multirow[t]{3}{*}{ Variables } & & \multirow{2}{*}{\multicolumn{2}{|c|}{ Grupo consumo }} & \multirow{2}{*}{\multicolumn{2}{|c|}{ Grupo no consumo }} \\
\hline & & & & & \\
\hline & & $N=25(\%)$ & $N=25(\%)$ & $N=25(\%)$ & $\mathrm{N}=25(\%)$ \\
\hline \multirow{3}{*}{$\begin{array}{l}\text { Consumo de alcohol } \\
\text { (dice de sí mismo) }\end{array}$} & No, nunca & $1(4)$ & $17(68)$ & $19(76)$ & $20(80)$ \\
\hline & En ocasiones & $16(64)$ & $8(32)$ & $6(24)$ & $4(16)$ \\
\hline & Con frecuencia & $8(32)$ & 0 & 0 & $1(4)$ \\
\hline \multirow[t]{3}{*}{ El otro consume alcohol } & No, nunca & $13(52)$ & 0 & $19(76)$ & $20(80)$ \\
\hline & En ocasiones & $10(40)$ & $1(4)$ & $3(12)$ & $5(20)$ \\
\hline & Con frecuencia & $2(8)$ & $24(96)$ & $3(12)$ & 0 \\
\hline \multirow[t]{3}{*}{ Tiene episodios de embriaguez } & Nunca & $9(36)$ & $24(96)$ & $24(96)$ & $25(100)$ \\
\hline & En ocasiones & $13(52)$ & $1(4)$ & $1(4)$ & 0 \\
\hline & Con frecuencia & $3(12)$ & 0 & 0 & 0 \\
\hline \multirow[t]{3}{*}{ Su pareja se embriaga } & Nunca & $23(92)$ & $1(4)$ & $23(92)$ & $25(100)$ \\
\hline & En ocasiones & $1(4)$ & $3(12)$ & $2(8)$ & 0 \\
\hline & Con frecuencia & $1(4)$ & $21(84)$ & & 0 \\
\hline \multirow[t]{3}{*}{ Violencia si embriaguez } & No, nunca & $19(76)$ & $25(100)$ & $25(100)$ & $24(96)$ \\
\hline & En ocasiones & 0 & 0 & 0 & $1(4)$ \\
\hline & Con frecuencia & $6(24)$ & 0 & 0 & 0 \\
\hline \multirow[t]{3}{*}{ Violencia cuando el otro bebe } & Nunca & $23(92)$ & 0 & $23(92)$ & $25(100)$ \\
\hline & En ocasiones & 0 & $2(8)$ & $2(8)$ & 0 \\
\hline & Con frecuencia & $2(8)$ & $23(92)$ & 0 & 0 \\
\hline \multirow{3}{*}{$\begin{array}{l}\text { Consumo de drogas } \\
\text { (dice de sí mismo) }\end{array}$} & No, nunca & $19(76)$ & $24(96)$ & $22(88)$ & $23(92)$ \\
\hline & En ocasiones & $5(20)$ & $1(4)$ & $3(12)$ & $2(8)$ \\
\hline & Con frecuencia & $1(4)$ & 0 & 0 & 0 \\
\hline \multirow[t]{3}{*}{ Consumo de drogas el otro } & No, nunca & $22(88)$ & $17(68)$ & $22(88)$ & $18(72)$ \\
\hline & En ocasiones & $2(8)$ & $4(16)$ & $3(12)$ & $6(24)$ \\
\hline & Con frecuencia & $1(4)$ & $4(16)$ & 0 & $1(4)$ \\
\hline \multirow[t]{2}{*}{ Ha ido a deshabituación } & Sí & $5(20)$ & & 0 & \\
\hline & No & $20(80)$ & & $25(100)$ & \\
\hline \multirow[t]{2}{*}{ Consumos del imputado } & Cocaína & $4(16)$ & 0 & $2(8)$ & 0 \\
\hline & Porros & $3(12)$ & 0 & $4(16)$ & 0 \\
\hline \multirow[t]{2}{*}{ El denunciado juega } & Sí & $4(16)$ & $10(40)$ & 0 & 0 \\
\hline & No & $21(84)$ & $15(60)$ & $25(100)$ & $25(100)$ \\
\hline
\end{tabular}

Tabla 6.

Análisis descriptivos y comparativos de los datos sobre consumo de sustancias adictivas en función del grupo. 
En la Tabla 7, con el cuestionario CAGE se observa que, en el grupo 1, el porcentaje de hombres que reconocen que tienen un problema relacionado con el consumo de alcohol es menor que el porcentaje que no lo admite. En la Tabla 8 destaca que un $40 \%$ de los hombres presenta sintomatología y signos físicos relacionados con el consumo de alcohol, como temblor de manos, insomnio, falta de apetito, pérdida de peso, fetor enólico, facies característica, etc.

En la Tabla 9 se comprueba que casi la mitad de la muestra admite que debería limitar sus ingestas de alcohol, que se sentiría más feliz si no bebiera y que su familia ha buscado ayuda. Aproximadamente un tercio de la muestra presenta fetor enólico y temblor de manos, y reconoce que el alcohol está destruyendo su vida y la relación con su pareja. Un porcentaje algo inferior (entre un $15 \%$ y un $25 \%$ ) presenta patología hepática, náuseas por la mañana, calma la resaca con alcohol, bebe antes del desayuno, bebe aunque se lo prohíba el médico, tolera grandes cantidades de alcohol e incluso no recuerda episodios posteriores a las ingestiones. Aún es menor el porcentaje de hombres que presenta patología grave relacionada con la ingesta de alcohol ( $8 \%$ a $15 \%$ ), como polineuropatía o delirium tremens, así como la necesidad de consumir alcohol para calmarse. Únicamente el $4 \%$ admite haber tenido problemas en el trabajo relacionados con sus consumos.

En la Tabla 10 se objetiva que cerca de la mitad de la muestra admite haber fracasado en sus intentos de dejar de beber. Un tercio bebe a pesar de los problemas que ello le causa. Entre un $15 \%$ y un $25 \%$ bebe más de lo que había planeado, dedica mucho tiempo a beber, bebe a pesar de saber que es perjudicial para su salud y que le causa problemas, e incluso se ha puesto en peligro por ello. Menos de un $15 \%$ admite que cada vez necesita beber más, que bebe para evitar los síntomas del síndrome de abstinencia y que ha tenido problemas legales relacionados con la bebida.

\begin{tabular}{|c|c|c|c|c|}
\hline \multirow[t]{3}{*}{ Variables } & \multicolumn{4}{|c|}{ Grupo consumo } \\
\hline & \multicolumn{2}{|c|}{ Sí } & \multicolumn{2}{|c|}{ No } \\
\hline & $\mathbf{N}$ & $\%$ & $\mathrm{~N}$ & $\%$ \\
\hline ¿Debe beber menos? & 16 & 64 & 9 & 36 \\
\hline ¿Le han criticado por beber? & 7 & 28 & 18 & 72 \\
\hline ¿Se siente culpable por ello? & 10 & 40 & 15 & 60 \\
\hline ¿Bebe por la mañana? & 3 & 12 & 22 & 88 \\
\hline
\end{tabular}

Tabla 8. Sintomatología.

\begin{tabular}{llcc}
\hline Variables & & Consumo & \% \\
\hline Tiene analítica reciente & & $\mathbf{N}=\mathbf{2 5}$ & 24 \\
& Sí & 6 & 76 \\
Sintomatología relacionada con consumo & No & 19 & 40 \\
& Sí & 10 & 60 \\
\cline { 2 - 3 } & No & $\mathbf{N}=10$ & $\%$ \\
& & 4 & 40 \\
\end{tabular}




\begin{tabular}{lcc}
\hline Variables & Sí & N \\
& $\%$ \\
\hline En los últimos 12 meses ha tomado 3 o más bebidas alcohólicas en 3 horas en 3 o más ocasiones & 16 & 64 \\
Cada vez necesita beber más para conseguir los mismos efectos & 3 \\
¿Bebe más para evitar los síntomas? & 3 & 12 \\
¿Bebe más de lo planteado? & 6 & 24 \\
¿Ha fracasado al tratar de reducir la ingesta? & 10 \\
¿Emplea mucho tiempo en conseguir alcohol, en beber y en recuperarse? & 40 \\
¿Pasa menos tiempo con amigos por beber? & 2 \\
¿Continuó bebiendo a pesar de las consecuencias en su salud? & 4 \\
¿Beber le ha causado problemas? & 6 & 16 \\
¿Ha bebido y se ha puesto en peligro? (conducir) & 6 & 24 \\
¿Ha tenido problemas legales por beber? & 4 & 16 \\
¿Continuó bebiendo a pesar de saber que le causaba problemas familiares? & 3 \\
¿En los últimos 12 meses tomó alguna sustancia de abuso en más de una ocasión? & 12 \\
\end{tabular}

Tabla 9. Cuestionario MINI.

\section{Discusión}

A continuación se explican los resultados obtenidos y se comparan con los de otros estudios.

En el estudio de O'Leary y Woodin ${ }^{27}$, en el que se realiza un análisis transversal de una muestra representativa, se objetiva una tendencia significativa entre la edad, la violencia hacia la pareja y el consumo de alcohol. Se produce una mayor prevalencia de consumo entre el principio y la mitad de la década de los 20 años, así como un importante descenso a medida que aumenta la edad (tanto en el consumo como en el empleo de la violencia). Dichos resultados son diferentes a los obtenidos en nuestro estudio, en el que la edad media del hombre consumidor es de 48 años (Tabla 1).

En lo que respecta a la raza, en el estudio de Stalans y Ritchie ${ }^{12}$ se nombran investigaciones, como la de Caetano ${ }^{28}$, que relacionan el consumo elevado de alcohol con el empleo de violencia fundamentalmente en la raza hispánica, pero no en la caucásica. Sin embargo, otras investigaciones, como la de Caetano ${ }^{29}$, muestran resultados dispares y ponen de manifiesto una relación de dicho consumo con la raza africana y americana, y escasa relación con la raza hispánica o caucásica. Por tanto, no se establece relación significativa entre el factor racial y el consumo de alcohol. En nuestro estudio, la mayoría de los hom- bres son caucásicos, y se valora en ellos una relación entre consumo de alcohol y aumento de la violencia ejercida (Tabla 1).

En el estudio de Riofrío y Rodríguez ${ }^{3}$ se enumeran una serie de factores epidemiológicos asociados a los trastornos relacionados con el alcohol, como pueden ser:

- El sexo: valoran que el consumo de alcohol es más frecuente en los varones que en las mujeres, a pesar de que en estas ha aumentado en los últimos años.

- La historia familiar: relacionan el desempleo o las profesiones en las que hay fácil acceso al alcohol y con estrés con una mayor probabilidad de consumo de alcohol.

- Factores psicológicos: relacionan los trastornos depresivos, ansiosos, del control de los impulsos y psicóticos con un mayor consumo de alcohol, pero consideran que no hay un perfil de personalidad predispuesta.

Como se ha visto en los resultados, los hombres del grupo de consumo presentan una mayor tasa de desempleo que los sujetos del grupo control, lo que puede explicarse por el menor nivel de estudios, que les permite acceder a una menor diversidad de posibilidades en el ámbito laboral. La elevada tasa de desempleo puede justificar, a su vez, que cerca 
Tabla 10 Cuestionario MALT

\begin{tabular}{|c|c|c|}
\hline \multirow[t]{2}{*}{ Variables } & \multicolumn{2}{|c|}{ Sí } \\
\hline & $N=25$ & $\%$ \\
\hline \multicolumn{3}{|l|}{ Cuestionario MALT-O } \\
\hline Patología hepática & 5 & 20 \\
\hline Polineuropatía & 3 & 12 \\
\hline Delirium tremens & 2 & 8 \\
\hline Aliento alcohólico & 8 & 32 \\
\hline $\begin{array}{l}\text { Consumo alcohólico superior a los } 150 \mathrm{ml} \text { (en la mujer, } 120 \mathrm{ml} \text { ) de } \\
\text { alcohol puro al día, al menos durante unos meses }\end{array}$ & 8 & 32 \\
\hline $\begin{array}{l}\text { Consumo alcohólico superior a los } 300 \mathrm{ml} \text { (en la mujer, } 240 \mathrm{ml} \text { ) de } \\
\text { alcohol puro, una o más veces al mes }\end{array}$ & 3 & 12 \\
\hline Familia ha buscado ayuda & 10 & 40 \\
\hline \multicolumn{3}{|l|}{ Cuestionario MALT-S } \\
\hline Me tiemblan las manos & 7 & 28 \\
\hline Tengo náuseas por la mañana & 5 & 20 \\
\hline Calmo la resaca bebiendo & 6 & 24 \\
\hline Me siento amargado por mis problemas & 18 & 72 \\
\hline Bebo antes del desayuno & 6 & 24 \\
\hline Necesito seguir bebiendo tras los primeros vasos & 3 & 12 \\
\hline Pienso en el alcohol a menudo & 4 & 16 \\
\hline Bebo aunque me lo prohíba el médico & 6 & 24 \\
\hline Si bebo más como menos & 7 & 28 \\
\hline Me han Ilamado la atención en el trabajo & 1 & 4 \\
\hline Prefiero beber solo & 3 & 12 \\
\hline Bebo de un trago y más deprisa que otros & 3 & 12 \\
\hline Desde que bebo más soy menos activo & 3 & 12 \\
\hline Me remuerde la conciencia & 7 & 28 \\
\hline He ensayado un sistema para beber & 4 & 16 \\
\hline Debería limitar mis ingestas & 10 & 40 \\
\hline Sin alcohol no tendría tantos problemas & 8 & 32 \\
\hline Bebo alcohol para calmarme & 3 & 12 \\
\hline Beber está destruyendo mi vida & 7 & 28 \\
\hline Cambio de idea respecto a dejar de beber & 6 & 24 \\
\hline Los demás no comprenden por qué bebo & 8 & 32 \\
\hline Si no bebiera me llevaría mejor con mi esposa & 8 & 32 \\
\hline He probado a pasar temporadas sin alcohol & 16 & 64 \\
\hline Sin alcohol estaría feliz conmigo mismo & 10 & 40 \\
\hline Me han mencionado mi aliento alcohólico & 6 & 24 \\
\hline Aguanto grandes cantidades & 5 & 20 \\
\hline No recuerdo lo ocurrido tras beber mucho & 5 & 20 \\
\hline
\end{tabular}


de la mitad de la muestra residan con sus padres u otros familiares. El hecho de que alrededor de un tercio de los sujetos del grupo que consume alcohol se consideren independientes, a pesar de que cerca de la mitad se encuentran en situación de desempleo, puede deberse a que dicha situación sea reciente y estén cobrando un subsidio por desempleo. Por el contrario, el alto porcentaje de hombres del grupo control que reside con sus padres a pesar de tener trabajo y ser económicamente independientes puede explicarse por el hecho de que, una vez rota la relación con la demandante, no tengan otro lugar donde poder vivir (Tabla 1 ).

Según el estudio de Stalans y Ritchie ${ }^{12}$, se establece una relación entre el consumo de sustancias de adicción y un bajo nivel socioeconómico. Igualmente relacionan el hecho de no querer seguir programas de tratamiento de violencia con no haber completado los estudios primarios o estar en situación de desempleo. Estos datos son en parte coincidentes con los de nuestro estudio, de manera que los sujetos del grupo de consumo presentan un menor nivel de estudios y una mayor tasa de desempleo (Tabla 1).

La mayoría de los hombres de la muestra del estudio realizado por Lipsky et al. ${ }^{14}$ presentan una media de edad elevada, son divorciados o separados, tienen un nivel de estudios elevado, pero una alta tasa de desempleo, y antes de comenzar el tratamiento provenían de un hogar temporal o carecían de hogar. Un $11,8 \%$ de los hombres ha sido arrestado por haber ejercido violencia hacia su pareja. Entre las mujeres de dicho estudio, más de un 5,2\% afirma haber sufrido malos tratos habituales y un $58 \%$ refiere haber sufrido violencia puntual por parte de su pareja. Relacionan los consumos con la violencia ejercida hacia la pareja. Los resultados de este estudio demuestran que los sujetos que únicamente beben pertenecen a la población nativa americana, de Alaska e hispánica de cualquier raza, mientras que, por el contrario, aquellos que beben y consumen drogas o padecen patología psiquiátrica pertenecen a la población blanca no hispánica. Como podemos comprobar al comparar estos resultados con los obtenidos en nuestro estudio, algunos de ellos son coincidentes, como la edad media elevada y la tasa de desempleo, entre otros.

Del análisis de los datos relacionados con la familia de referencia se valora que el hecho de que los padres de un número mayor de hombres en el grupo de consumo hayan ejercido malos tratos hacia sus mujeres (madres de los imputados), y que hayan consumido alcohol y sustancias de adicción en mayor proporción, ha podido suscitar un comportamiento imitativo en los imputados, quienes a su vez emplean el alcohol y la violencia como modo de solventar sus problemas o como modo de relacionarse con sus parejas, aprehendiendo e interiorizando tales conductas (Tabla 2).

De los datos relativos a las relaciones de pareja se objetiva que el hecho de que dos hombres del grupo de consumo sigan manteniendo relación con las denunciantes y uno conviva con ella puede ser indicativo de falta de aceptación de la ruptura y negación a abandonar el domicilio conyugal. Igualmente puede ser indicativo de que la denunciante haya decidido retirar la denuncia, bien voluntariamente, bien bajo presión o chantaje emocional por parte del imputado, o como consecuencia de la dependencia que sufren algunas mujeres; de ser así, dicha dependencia de la denunciante o el control por parte del imputado estarían presentes en mayor medida en el grupo de consumo de alcohol (Tabla 2).

En lo que a los posibles motivos de discusión se refiere (Tabla 3), vemos que la actitud de la pareja o el comportamiento celoso es una de las principales causas, siendo superior en el grupo de consumo. Esto podría explicarse porque el consumo de alcohol supone, como ya se ha expuesto, una mayor desinhibición en la conducta, lo que facilita a su vez dicho comportamiento celoso. Según viene indicado en el estudio de Stalans y Ritchie ${ }^{12}$, el consumo de alcohol genera suspicacia, interpretaciones erróneas y sospechas de infidelidades, lo que junto con un posible deterioro en la potencia sexual del maltratador va a suponer una actitud violenta en el consumidor. En dicho estudio se afirma que el consumo de alcohol conlleva un mayor gasto económico y de tiempo, lo que supone un motivo de discusión en la pareja, al igual que ocurre en nuestro estudio (Tabla 3). A su vez, los conflictos generados en una pareja con un nivel socioeconómico bajo suponen una escalada en la violencia, debido a la limitada fuente de ingresos disponible para gastar en alcohol cuando las necesidades básicas apenas pueden cubrirse.

Centrándose en el estudio de los malos tratos ejercidos por el hombre hacia su pareja (Tabla 3), el hecho de que más hombres del grupo de consumo que del grupo control empleen violencia contra sus parejas puede deberse a que el consumo de alcohol entabla o genera a su vez mayor probabilidad de empleo de violencia verbal y física, tal como se afirma en el estudio de Stalans y Ritchie ${ }^{12}$.

Que los hijos estén presentes en gran parte de las conductas de violencia ocurridas (Tabla 3 ) es un factor de riesgo para aprender dicho comportamiento e interiorizarlo como apropiado. De ser así, la violencia que el imputado ha vivido en casa, presenciada en 
las conductas que su padre ejercía hacia su madre, va a ser transmitida a la una nueva generación, como ya se ha comentado.

En la Tabla 6 se ha tratado de diferenciar si el consumo de alcohol es el causante de la violencia o si supone un aumento en la agresividad de base en el grupo demandado. Resulta destacable que gran parte de las mujeres del grupo de consumo afirman de sus parejas, durante la exploración, que únicamente ejercen violencia contra ellas cuando se encuentran bajo los efectos del alcohol. Sin embargo, cuando se analizan las conductas violentas individualmente se objetiva que en realidad la violencia se produce con mayor frecuencia cuando el imputado no ha bebido. Es compatible que las denunciantes traten de justificar en tales consumos la violencia que ejercen sus parejas, por lo doloroso que puede resultarles el hecho de admitir que están sufriendo malos tratos. Al comparar ambos grupos (consumo y control) se observa que las conductas de malos tratos son mayores en el grupo que consume alcohol, lo que puede suponer que tal hábito aumente la violencia ejercida por parte del imputado, pero no es la única causa del empleo de la violencia, tal como se ha explicado en la introducción del presente estudio.

En relación con lo expuesto en el párrafo anterior, en el estudio de Lee et al. ${ }^{30}$ el $70 \%$ de las víctimas afirman que sus parejas estaban bajo la influencia del alcohol o de otras sustancias de adicción en el momento en que fueron agredidas. Dichos autores valoran que las personas agresivas se excusan con el consumo de alcohol o justifican su comportamiento afirmando que "el alcohol genera agresividad". A pesar de que la literatura consultada para realizar tal investigación recoge que los agresores que consumen alcohol son más propensos a emplear violencia contra sus parejas que aquellos que no lo consumen, los resultados muestran que no hay diferencias en cuanto al consumo de alcohol entre los hombres que agreden a sus parejas y aquellos que no lo hacen, por lo que el consumo de tal sustancia no justifica su comportamiento agresivo. Tales resultados distan de los obtenidos en nuestro estudio, en los que sí hay diferencia, en cuanto al empleo de la violencia, entre los hombres que beben alcohol y aquellos que no lo hacen.

En la revisión realizada por Foran y O'Leary ${ }^{31}$ se objetiva una escasa o nula asociación entre el consumo de alcohol y la violencia ejercida contra la pareja en algunos estudios, y una asociación moderada en otros de los estudios analizados, dependiendo del tipo de muestra estudiada, del modo en que se valora la agresión y del tipo de medida o la forma de cuantificar el consumo de alcohol (incluye frecuencia y cantidad).
Afirman que, a pesar de que estudios experimentales en el laboratorio demuestran una relación entre consumo de alcohol y respuestas agresivas, para sorpresa de muchos esta asociación es controvertida. Se expone que en ocasiones se emplea el consumo de alcohol como modo de restar importancia o justificar una conducta, o para tratar de demostrar la inimputabilidad del autor de la agresión. Igualmente, la mujer se excusa con dicho consumo como manera de perdonar con mayor facilidad el comportamiento de su pareja ("realmente no quería") y retomar una relación potencialmente peligrosa. A pesar de lo expuesto anteriormente, hay estudios que demuestran que el tratamiento para la reducción de alcoholismo supone una disminución del empleo de la violencia.

Dentro de la revisión de Foran y O'Leary ${ }^{31}$, los trabajos de Graham et al. ${ }^{32}$, Leonard ${ }^{33}$ y Lipsey et al. ${ }^{34}$ afirman que la asociación entre alcohol y agresión se debe a otros factores o variables, como la edad joven, las formas anormales de comportamiento, los conflictos en la relación de pareja, la insatisfacción, y factores de provocación o frustración, entre otros. Sin embargo, cuando dichas variables son controladas, el estudio pone de manifiesto que se mantiene la asociación entre consumo de alcohol y empleo de violencia hacia la pareja. Pero sí hay que tener en cuenta que dichos factores, junto con las características o los rasgos de personalidad de cada hombre, suponen que el consumo de alcohol se relacione con el empleo de agresividad en algunos y no en otros. Los resultados de su estudio muestran una significativa, pero moderada, asociación entre el consumo de alcohol y la agresión. Se plantea la hipótesis de que cuantos más factores de riesgo o variables presente el sujeto mayor será la asociación entre el consumo de alcohol y el empleo de la violencia.

El hecho de que los hombres de ambos grupos consideren que sufren malos tratos puede deberse a que traten de presentarse como víctimas ante el proceso judicial, minimizando o negando así sus conductas al estar siendo explorados; o por el contrario, puede ocurrir que dicha violencia sea cierta, o que sea empleada por la mujer como medio de defensa (Tabla 3). Según el estudio de O'Leary y Woodin²7, el empleo de la violencia física por parte del hombre es más frecuente y grave que en la mujer, y con peores consecuencias o daños. Del mismo modo, valoran que el problema de abuso del alcohol es mayor en los hombres que en las mujeres. Estos datos son coincidentes con los de nuestro estudio.

De los resultados de la Tabla 5 podría interpretarse que el mayor número de hombres del grupo de consumo que presentan patología física y mental podría ser consecuencia del propio consumo de alcohol, 
que ha generado algunos de los síntomas médicos y psiquiátricos vistos durante la introducción. Según Lipsky et al. ${ }^{14}$, entre los hombres que consumen alcohol de manera abusiva, en los Estados Unidos, es más frecuente que se produzcan patología mental (con una prevalencia del $44 \%$ ) y consumo de otras sustancias de adicción (36\%), a lo que denominan "COD" (co-ocurring substance abuse and mental disorders). Dichos individuos, a su vez, experimentan patología somática crónica y son usuarios frecuentes de los servicios sanitarios en mayor proporción que el resto de la población. Estos autores consideran el consumo de alcohol y de otras sustancias de abuso como predictores importantes o factores de riesgo asociados a violencia en la pareja.

Del análisis de los datos sobre las posibles denuncias y conductas delictivas (Tabla 5) se desprende que es mayor el número de mujeres del grupo de consumo que han retirado la denuncia, lo que puede ser de nuevo un indicativo de la dependencia emocional que sufren hacia sus parejas, o de la presión o las amenazas recibidas por parte de ellos para conseguir tal fin. El hecho de que un mayor número de hombres del grupo de consumo tenga antecedentes penales puede ser indicativo de que cuando el sujeto se encuentra bajo los efectos del alcohol es más proclive a cometer actos delictivos.

El estudio de Oberleitner et al. ${ }^{15}$ fue realizado en una muestra de 73 hombres con dependencia del alcohol, detenidos por haber ejercido violencia contra sus parejas y que realizaban una terapia cognitivoconductual. En su estudio, tal como se ha visto en la introducción, y al igual que ocurre en nuestra investigación, se establece una relación entre el consumo de alcohol y el aumento del empleo de violencia, en personas agresivas de base, en las que el alcoho actúa como "exacerbador" de la violencia.

En la Tabla 6, en la que se muestran los datos sobre consumo de sustancias adictivas, se observa que, a pesar de que un cierto porcentaje de hombres admite que bebe, pocos son realmente conscientes de su problema, de manera que únicamente un escaso porcentaje trata de abandonar tal hábito y acude a tratamientos de desintoxicación-deshabituación. Igualmente se comprueba en dicha tabla el escaso, pero existente, porcentaje de mujeres que reconoce que bebe alcohol.

Si comparamos nuestro estudio con otros, como el de Wilson-Cohn et al. ${ }^{35}$, se obtiene que en él de estos autores un $48 \%$ de las mujeres de la muestra de Portland y un $37 \%$ de la muestra de las mujeres de Nueva York afirman que su propio consumo de sustancias de adicción ha contribuido o ha estado relacionado con los conflictos 0 agresiones sufridas por sus parejas. Igualmente, tanto las mujeres de Portland (68\%) como las de Nueva York (50\%) afirman que el consumo de sustancias de adicción por parte de sus parejas ha estado relacionado con los conflictos o agresiones sufridas durante su relación.

En la investigación realizada por Fals-Stewart et al. ${ }^{4}$, en la que se estudian parejas en las que el hombre consume sustancias de adicción, se ha encontrado una relación entre dicho consumo y los problemas en las relaciones de pareja, los cuales mejoran cuando se trata la adicción, no sólo de manera individual, sino sobre todo cuando ambos miembros de la pareja reciben terapia para la adicción. La mayoría de los sujetos de nuestro estudio no han seguido tratamientos de desintoxicación-deshabituación (Tabla 6).

A partir de las Tablas 6 a 8 puede interpretarse que un cierto porcentaje de hombres, inferior a la mitad en su mayoría, admite cierto consumo de alcohol y problemas derivados de este (físicos, laborales, relacionales, etc.). A medida que la gravedad de la sintomatología y de los problemas derivados de tal consumo aumenta, es inferior el porcentaje de hombres que responden de manera afirmativa a las cuestiones. Es compatible que sea cierto que únicamente un escaso porcentaje presenta un importante deterioro físico y un elevado consumo, pero igualmente podría explicarse por una posible minimización de los consumos al encontrarse en un medio judicial, por las repercusiones que implicaría admitirlo.

\section{Conclusiones}

- En relación con el objetivo 1: del estudio de las conductas violentas en ambos grupos se desprende que hay notables diferencias entre ellos en lo que a la frecuencia del empleo de conductas violentas se refiere. De los resultados se obtiene que es mayor el porcentaje de conductas de malos tratos ejercidas por los hombres del grupo que consumen alcohol.

- En relación con el objetivo 2: del estudio de las conductas violentas en el grupo de hombres que han sido denunciados por consumo de alcohol se desprende que es mayor la frecuencia de la violencia ejercida en la mayoría de los ítems estudiados cuando los hombres no han consumido alcohol. La frecuencia de dicha violencia, por el contrario, es menor cuando beben, pero se suma a la violencia ya ejercida en estado sobrio.

- En relación con la hipótesis 1: tal como se ha comentado en el objetivo 1 , se cumple la hipó- 
tesis de que hay relación entre el consumo de alcohol y el aumento del empleo de la violencia, pero no de manera directa. Es decir, el consumo de alcohol no explica por sí mismo el empleo de violencia contra la pareja, sino que actúa como un factor que participa en ella.

- En relación con la hipótesis 2: no se cumple la hipótesis. Los hombres que consumen alcohol y son violentos ejercen violencia en igual o mayor medida cuando no se encuentran bajo los efectos del alcohol. El consumo de alcohol supone una desinhibición en su conducta violenta de base, la cual aumenta tras los consumos, produciéndose un efecto "sumatorio" o un incremento de tal violencia.

- En relación con la hipótesis 3: se cumple la hipótesis. Los resultados muestran que los hombres que no consumen alcohol van a ejercer violencia con menor frecuencia hacia sus parejas, y son capaces de controlar su ira, impulsividad o agresividad, por tener intactos los mecanismos inhibidores en ausencia de alcohol.

Los autores declaran no tener ningún conflicto de intereses.

\section{Bibliografía}

1. Ministerio del Interior. Encuesta domiciliaria sobre el consumo de drogas. Madrid: Delegación de Gobierno para el Plan Nacional sobre Drogas; 2004.

2. Manual Diagnóstico y Estadístico de las Enfermedades Mentales. Versión española de la cuarta edición revisada de la obra original en lengua inglesa publicada por la Asociación de Psiquiatría Americana. Barcelona: Masson; 2005.

3. Riofrío C, Rodríguez JJ. Identificación del alcoholismo en atención primaria. Jano: Medicina y Humanidades. 2010; 65-72.

4. Fals-Stewart W, Birchler GR, Ellis L. Procedures for evaluating the dyadic adjustment of drug-abusing patients and their intimate partners. Journals of Substance Abuse Treatment. 1999;16:5-16.

5. Zaczyk C. La agresividad, comprenderla y evitarla. Barcelona: Paidós; 2005.

6. Chermack ST, Taylor S. Alcohol and human physical aggression: pharmacological versus expectancy effects. Journal of Studies on Alcohol. 1995;56:44956.

7. López F, Álamo C. Historia de la psicofarmacología. Madrid: Médica Panamericana; 2007.

8. Bell KM, Naugle AE. Intimate partner violence theoretical considerations: moving towards a contextual framework. Clinical Psychology Review. 2008;28:1096-107.

9. Margolin G, Burman B. Wife abuse versus marital violence: different terminologies, explanations and solutions. Clinical Psychology Review. 1993;13:5973.
10. Lee WV, Gottheil E, Sterling RC, Weinstein SP, Serota RD. Characteristics of cocaine-addicted individuals who abuse their partners. Journal of Substance Abuse Treatment. 1997;14:367-71.

11. Keiley MK, Keller PS, El-Sheikh M. Effects of physical and verbal aggression, depression and anxiety on drinking behavior of married partners: a prospective and retrospective longitudinal examination. Aggressive Behavior. 2009;35(4):296-312.

12. Stalans LJ, Ritchie J. Relationship of substance use/abuse with psychological and physical intimate partner violence: variations across living situations. Journal of Family Violence. 2008;23:9-24.

13. Lorente M, Toquero de la Torre F. Guía de la buena práctica clínica en abordaje en situaciones de violencia de género. Madrid: International Marketing and Comunications; 2004.

14. Lipsky S, Krupski A, Roy-Byrne P, Lucenko B, Mancuso D, Huber A. Effect of co-occurring disorders and intimate partner violence on substance abuse treatment outcomes. Journal of Substance Abuse Treatment. 2010;38:231-44.

15. Oberleitner LMS, Mandel DLCSW, Easton CJ. Treatment of co-occurring alcohol dependence and perpetration of intimate partner violence: the role of anger expression. Journal of Substance Abuse Treatment. 2013;45:313-8.

16. Bennett LW. Substance abuse by men in partner abuse intervention programs: current issues and promising trends. Violence and Victims. 2008;23:236-48. 
17. Hines DA, Douglas EM. Alcohol and drug abuse in men who sustain intimate partner violence. Aggressive Behavior. 2012;38(1):31-46.

18. Kantor GK, Straus MA. The "drunken bum" theory of wife beating. En: Straus MA, Gelles RJ, editors. Physical violence in American families: risk factors and adaptations to violence in 8,145 families. New Brunswick, NJ: Transaction Publishers; 1990. p. 203-334.

19. Miller BA. Partner violence experiences and women's drug use: exploring the connections. En: Wetherington $\mathrm{CL}$, Roman $\mathrm{AB}$, editors. Drugs addiction research and the health of women. Washington, DC: National Institute on Drug Abuse; 1998. NIH Publication No. 98-4290. p. 407-16.

20. Testa M, Livingston JA, Leonard KE. Women's substance use and experiences of intimate partner violence: a longitudinal investigation. Addictive Behaviors. 2003;28:1649-64.

21. Hirschel D, Hutchison IW. The interrelationship between substance abuse and the likelihood of arrest, conviction and re-offending in cases of intimate partner violence. Journal Family Violence. 2010;25:8190.

22. Cunradi CB, Ames GM, Duke M. The relationship of alcohol problems to the risk for unidirectional and bidirectional intimate partner violence among a sample of blue-collar couples. Violence and Victims. 2011;26(2):147-58.

23. Klostermann $\mathrm{KC}$, Fals-Stewart W. Intimate partner violence and alcohol use: exploring the role of drinking in partner violence and its implications for intervention. Aggression and Violent Behavior. 2006;11:587-97.

24. Leonard KE. Alcohol and intimate partner violence: when can we say that heavy drinking is a contributing cause of violence? Addiction. 2005;100:422-5.

25. Caetano R, Ramisetty-Mikler S, Field CA. Unidirectional and bidirectional intimate partner violence among white, black, and Hispanic couples in the United States. Violence and Victims. 2005;20(4):393-405.
26. Downs WR, Miller BA, Panek DD. Differential patterns of partner-to-woman violence: a comparison of samples of community, alcohol-abusing, and battered women. Journal of Family Violence. 1993;8:113-35.

27. O'Leary KD, Woodin, EM. Partner aggression and problem drinking across the lifespan: how much do they decline? Clinical Psychology Review. 2005;25:877-94.

28. Caetano R, Cunradi C, Schafer J, Clark C. Intimate partner violence and drinking patterns among White, Black and Hispanic couples in the U.S. Journal of Substance Abuse. 2000;11:123-38.

29. Caetano R, Schafer J, Cunradi CB. Alcohol-related intimate partner violence among White, Black, and Hispanic Couples in the United States. Alcohol Research \& Health. 2001;25(1):58-65.

30. Lee WV, Gottheil E, Sterling RC, Weinstein SP Serota RD. Characteristics of cocaine-addicted individuals who abuse their partners. Journal of Substance Abuse Treatment. 1997;14 (4):367-71.

31. Foran H, O'Leary KD. Alcohol and intimate partner violence: a meta-analytic review. Clinical Psychology Review. 2008;28:1222-34.

32. Graham K, Wells S, West P. A framework for applying explanations of alcohol-related aggression to naturally occurring aggressive behavior. Contemporary Drug Problems. 1997;24(4):625-66.

33. Leonard KE. Domestic violence: What is known and what do we need to know to encourage environmental interactions. Journal of Substance Use. 2001;6:235-47.

34. Lipsey MW, Wilson BM, Cohen MA, Derzon JH. Is there a causal relationship between alcohol use and violence? New York: Plenum Press; 1997.

35. Wilson-Cohn C, Strauss SM, Falkin GP. The relationship between partner abuse and substance use among women mandated to drug treatment. Journal of Family Violence. 2002;17(1):91-105. 